\title{
新型三唑桥联杂化复式环糊精手性固定相的构筑及性能研究
}

\author{
张丽芳 ${ }^{a, b}$ 赵杰 ${ }^{c}$ 王勇 $*, a, b$ \\ $\left({ }^{a}\right.$ 天津大学理学院化学系 天津 300072) \\ ( ${ }^{b}$ 天津化学化工协同创新中心 天津 300072$)$ \\ ( ${ }^{c}$ 天津市农业资源与环境研究所 天津市农业科学院 天津 300192)
}

\begin{abstract}
摘要 运用 $\mathrm{Cu}(\mathrm{I})$ 催化的 1,3-偶极环加成反应(点击化学), 以乙酸酩修饰单叠氮环糊精为底层, 炔基衍生的天然环糊精 为顶层，在硅胶表面通过自下而上的方式构建了一种新型 “天然一乙酰基衍生化” 三唑桥联杂化复式环糊精手性固定相 材料(ANCDCSP)，并通过红外光谱、热重和元素分析等测试手段对其结构进行了表征. 该固定相材料可提供包合作用、 氢键给体、氢键受体、偶极-偶极作用等多重识别位点以及底层和顶层环糊精间的协同效应. 在 HPLC 反相分离模式下, 该固定相材料对 10 种丹磺酰氨基酸类(Dns amino acids)、10 种小分子芳香酸(phenyl carboxylic acids)、5 种异噁唑啉类 (isoxazolines)以及多种其它手性对映体具有良好的手性拆分效果. 其中 Dns-Phe、DL-3PhLacA 以及 Bendroflumethiazide 的分离度分别达到 5.3、4.1 和 4.1, 且多数手性对映体的分离度优于课题组先前制备的复式天然环糊精手性固定相 (DCDCSP).
\end{abstract}

关键词＼cjkstart点击化学；复式环糊精；手性固定相；手性识别；高效液相色谱

\section{Construction and Chromatographic Performance of a Novel Triazole Bridged Hybrid Bilayer Cyclodextrin Chiral Stationary Phase}

\author{
Zhang, Lifang ${ }^{a, b} \quad$ Zhao, Jie $^{c} \quad$ Wang, Yong*,a,b \\ ( ${ }^{a}$ Department of Chemistry, School of Science, Tianjin University, Tianjin 300072) \\ ( ${ }^{b}$ Collaborative Innovation Center of Chemical Science and Engineering, Tianjin 300072) \\ ( ${ }^{c}$ Tianjin Academy of Agricultural Sciences, Tianjin 300192)
}

\begin{abstract}
A novel tandem-inverted triazole-bridged duplex "native-acetylated" hybrid cyclodextrin (CD) stationary phase material (ANCDCSP) was constructed via a surface-up 'click' approach. Mono-6-azido-CD was first immobilized onto the pre-dried silica surfaces as the down layer via ether linkage on $\mathrm{C} 2$ position, followed by acetylation of the cyclodextrin $-\mathrm{OH}$ groups with acetic anhydride. The top layer was fabricated by anchoring the synthesized alkyne functionalized CD onto the down $\mathrm{CD}$ layer via organic soluble $\mathrm{Cu}(\mathrm{I})$ catalytic 1,3-dipolar cycloaddition reaction (click chemistry) reported by us previously. The obtained crude material was purified by washing with $N, N$-dimethylformamide (DMF) twice followed by Soxlet extraction with acetone to afford the novel triazole-bridged duplex hybrid CD CSP and its structure was characterized via Fourier Transfer Infrared Spectroscopy (FTIR), thermogravimetric analysis (TG) and elemental analysis (EA). The hybrid bilayer ANCDCSP can provide multiple interaction sites such as $\mathrm{H}$-bonding $(\mathrm{OH}, \mathrm{C}=\mathrm{O}, \mathrm{NH})$, steric effects, dipole-dipole and synergistic effect inclusion complexation, which helps to broaden the CSP's enantioselectivity profile and enhance the enantioselectivity to some specific analytes. The CSP was applied for HPLC enantioseparation of various chiral compounds such as dansyl amino acids, carboxylic aryl acids, isoxazolines and some other racemates under reversed-phase separation mode using methanol/water and methanol/buffer as the mobile phases. Most of the dansyl amino acids and carboxylic aryl acids as well as bendroflumethiazide can be baseline separated. The isoxazolines, phenyl oxirane, atropine, $D L$-norleucine methyl ester and benfluorex were partially separated. The resolutions of Dns-Phe, $D L-3 \mathrm{PhLacA}$ and Bendroflumethiazide reached 5.3, 4.1 and 4.1, respectively. The separation ability of the current CSP is superior to that of the duplex native cyclodextrins chiral stationary phase (DCDCSP) prepared by our group previously, especially in separation of dansyl amino acids, isoxazolines and bendroflumethiazide. Besides, the current CD CSP affords relatively good stability, which was verified by the satisfied reproducibility after 150 runs with or without buffer $(\mathrm{pH} 4.10)$.

Keywords click chemistry; bilayer cyclodextrin; chiral stationary phase; enantiorecognition; HPLC ester
\end{abstract}

\footnotetext{
*E-mail: wangyongtju@tju.edu.cn

Received August 3, 2015; published September 14, 2015.

Supporting information for this article is available free of charge via the Internet at http://sioc-journal.cn.

Project supported by the National Natural Science Foundation of China (No. 21205086), Natural Science Foundation of Tianjin, China (No. 13JCQNJC05400) and the Independent Innovation Foundation of Tianjin University (No. 2015XRX-0021).

项目受国家自然科学基金(No. 21205086)、天津市自然科学基金(No. 13JCQNJC05400)、天津大学自主创新基金(No. 2015XRX-0021)资助.
} 


\section{1 引言}

在现代医药、农药及食品添加剂领域中, 手性药物 已成为当前医药领域的主要研究方向之一 ${ }^{[1,2]}$. 许多药 物的两个对映异构体在体内的药理活性、代谢过程、速 率及毒性等差异显著, 单一构象手性药物的需求及对药 物药理和毒理的关注与日剧增 ${ }^{[3,4]}$. 因此, 手性分离成为 当前研究的热点和难点 ${ }^{[5]}$. 在众多的手性分离技术中, 应用手性固定相(CSPs)的色谱分析分离技术已被广泛 应用于手性药物生产在线分析以及单一对映体药物的 规模制备, 成为迄今为止应用最为成功的分离手段之 一 [6]. 其中, 环糊精手性固定相以其优异的手性选择性 以及良好的化学稳定性始终处在该研究领域的前沿 ${ }^{[7]}$.

环糊精(Cyclodextrin, CD) 是一种具有特殊疏水空腔 的非还原性天然寡糖, 能与多种无机或有机分子形成稳 定的主体-客体包合物, 且多个手性 $\mathrm{C}$ 原子构成了良好 的不对称环境, 使其在分离对映体方面取得一系列成 果 ${ }^{[8]}$. 目前应用广泛的大都是基于天然或改性环糊精的 单一型手性固定相以及于新型功能键合臂的开发, 虽然 制备出了一系列优秀的单层环糊精手性固定相, 但是性 能优异的新型环糊精固定相的数目正在减少, 这成为手 性分离技术发展的瓶颈 ${ }^{[9]}$. 近年来, 桥联环糊精二聚体 受到广泛的关注, 与普通的环糊精相比, 桥联环糊精二 聚体可以通过两个邻近环糊精单元的协同作用而对客 体分子具有更强的包合能力, 为了使环糊精双分子层能 更好地发挥协同作用, 不同结构环糊精二聚体的合成成 为一个热点研究领域 ${ }^{[10]}$.

另一方面, “点击化学” 是端炔基与叠氮基经过 1,3偶极环加成反应而生成三唑桥环, 具有高选择性、高转 化率、反应后处理及产物分离简单方便的特点 ${ }^{[1]}$. 在固 定相键合过程中, 点击化学反应不会导致功能分子的结 构和功能的变化, 从而保证了分离材料的选择性. 因此 多个研究小组把点击化学技术用于制备色谱分离材料, Liang 课题组和 $\mathrm{Ng}$ 课题组在将 “点击”技术应用到环糊 精固定相的制备方面作出了突出贡献. 到目前为止, 运 用该方法已成功将环糊精及其衍生物通过单三唑键连 接到硅胶表面从而获得了不同类型的结构可控的 $\mathrm{CD}-\mathrm{CSP}^{[12]}$. 因此, $\mathrm{Cu}(\mathrm{I})$ 催化的点击化学方法为制备新 型杂化复式环糊精手性色谱材料提供了一种优良的途 径, 也为固体基质表面的功能化方法提供了有益参 考 ${ }^{[13,14]}$.

我们课题组前期已经运用 $\mathrm{Cu}(\mathrm{I})$ 催化的 1,3-偶极环 加成反应(点击化学), 在多孔硅胶表面自上而下地构建 复式环糊精双分子层, 制备了一种反向串联天然环糊精 固定相材料(DCDCSP), 并实现了丹磺酰氨基酸和小分 子芳香酸的有效拆分 ${ }^{[15]}$. 为了进一步拓展该类新型固 定相材料, 进一步提升该类材料的拆分能力, 本文通过 “点击” 技术在硅胶表面自下而上的构建了复式杂化环 糊精双分子层, 制备了新型杂化复式环糊精固定相
(ANCDCSP). 通过对底层环糊精进行乙酸酐改性引入 氢键和偶极 - 偶极作用, 结合三唑桥及环糊精空间的层 间协同效应，这种杂化复式环糊精固定相对手性对映体 具有更强的包合作用和手性识别能力. 这种逐层固载技 术也为开发基于环糊精二聚体的新型功能材料提供了 切实可行的方法.

\section{2 结果与讨论}

\subsection{ANCDCSP 的制备与表征}

ANCDCSP 的制备主要经历 $\mathrm{N}_{3} \mathrm{CD}$ 的固载、酯化以 及与炔基环糊精的点击化学反应. 因此本文通过红外光 谱、热重及元素分析对反应产物进行了表征.

$\mathrm{N}_{3}$ CDCSP 的红外谱图在 $2112 \mathrm{~cm}^{-1}\left(\mathrm{~N}_{3}\right.$ 基团)和 $2935 \mathrm{~cm}^{-1}$ (CD 上亚甲基)的吸收峰说明 $\mathrm{N}_{3}-\mathrm{CD}$ 已经成功 地固载到硅胶上(图 1). 单层乙酰基衍生化环糊精固定 相(ACDCSP)的红外谱图在 $1750 \mathrm{~cm}^{-1}$ 处的吸收峰说明 乙酰基的成功取代, 点击反应之后, $2112 \mathrm{~cm}^{-1}$ 处的吸收 峰几乎完全消失，证明 $\mathrm{N}_{3}$ 基团通过与炔丙氨基- $\mathrm{CD}$ 的 反应转化率较高. 由室温升到 $700{ }^{\circ} \mathrm{C}$, 升温速率 $10{ }^{\circ} \mathrm{C} /$ $\min$, ACDCSP 和 ANCDCSP 的热失重分别为 $23.1 \%$ 和 $25.4 \%(W / W)$ (图 S1). 元素分析结果表明, $\mathrm{N}_{3} \mathrm{CD}$-silica 依 次经过乙酰基衍生化和点击制备反应后生成的 ACDCSP 和 ANCDCSP 碳含量分别为 $8.32 \%$ 和 $11.05 \%$, 氢含量分别为 $1.59 \%$ 和 $1.98 \%$, 均有明显上升(表 S1). 由 于空间位阻的影响，存在部分没有形成复式环糊精双分 子层的部分，根据反应前后元素含量的变化，可以估算 得到其中的双分子层结构约占 $72 \%$. 红外光谱、热重及 元素分析都表明环糊精双分子层在硅胶表面的成功构 建, 且在课题组之前的研究中已证明底层环糊精的大口 端羟基与硅烷偶联剂进行亲核反应，因此在硅胶表面得

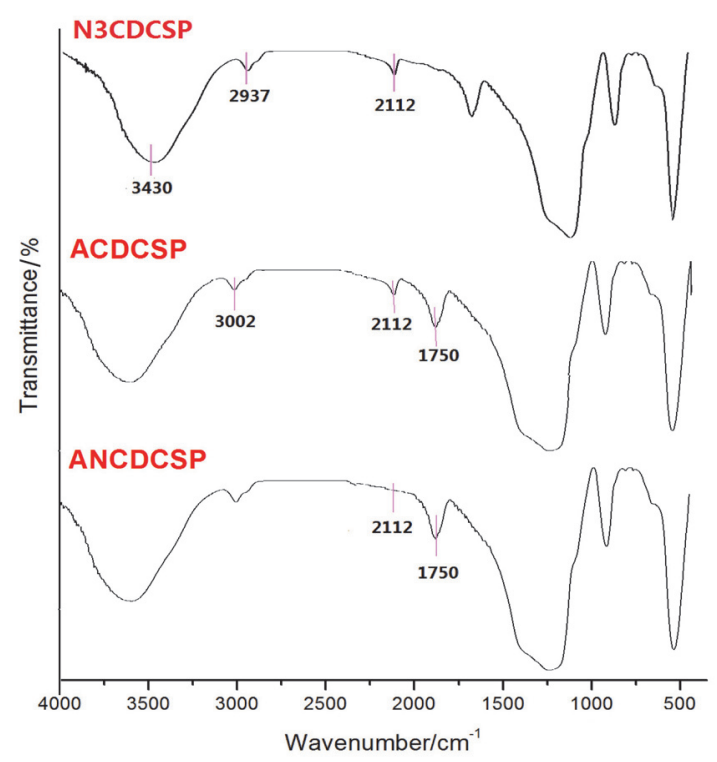

图 $1 \mathrm{~N}_{3} \mathrm{CDCSP}$ 与 $\mathrm{ANCDCSP}$ 的红外光谱 Figure 1 FTIR of $\mathrm{N}_{3} \mathrm{CDCSP}$ and DCDCSP 
到的是反向串联的环糊精双分子层 ${ }^{[15]}$. 且反应前后硅 胶颗粒没有明显破损(图 S2).

\subsection{ANCDCSP 手性分离性能的考察}

为全面考察所制备的新型固定相材料的手性拆分 性能, 本文选取 10 种丹磺酰氨基酸(Dansyl amino acids)、10 种小分子芳香酸(Aryl carboxylic acids)、5 种异 噁唑啉(isoxazolines)、苄氟噻嗪(Bendroflumethiazide)、

环氧苯乙烷(Phenyl oxirane)、阿托品(Atropine)、己氨酸 甲基酯 $(D L$-norleucine methyl ester) 以及苯氟雷司 (Benfluorex)等 30 多种对映异构体进行其拆分行为研究, 对映异构体结构如图 S3 所示.

\subsection{1 丹磺酰氨基酸的拆分}

丹磺酰氨基酸类化合物被广泛应用于考察环糊精 (特别是天然环糊精) 固定相材料的常用对映异构体, 本 文选取了丹磺酰戊氨酸(Dns-Nva)、丹磺酰缬氨酸 (Dns-Val)、丹磺酰亮氨酸(Dns-Leu)、丹磺酰谷氨酸 (Dns-Glu)、丹磺酰丁氨酸(Dns-Aba)、丹磺酰辛氨酸 (Dns-Aca)、丹磺酰正亮氨酸(Dns-Nle)、丹磺酰苯丙氨 酸(Dns-Phe)、丹磺酰丝氨酸(Dns-Ser)、丹磺酰苏氨酸 (Dns-Thr)等 10 种氨基酸, 以甲醇和缓冲液为流动相进 行固定相的色谱性能考察, 其分离结果如表 1 所示.

由表 1 可知, 所制备的杂化复式环糊精固定相对所 有的丹磺酰氨基酸都具有良好的分离效果, 其中 Dns-Nva、Dns-Val、Dns-Leu、Dns-Aca、Dns-Phe、Dns-Nle 六个样品都实现基线分离, Dns-Phe 的分离度高达 5.3. 这主要是因为分析物分子中的丹磺酰基团与环糊精的 疏水空腔的大小比较契合, 形成较好的 “主一客体” 络合 物后, 与手性碳相连的侧链基团可与另一环糊精空腔产 生匹配的疏水立体作用.

(a)

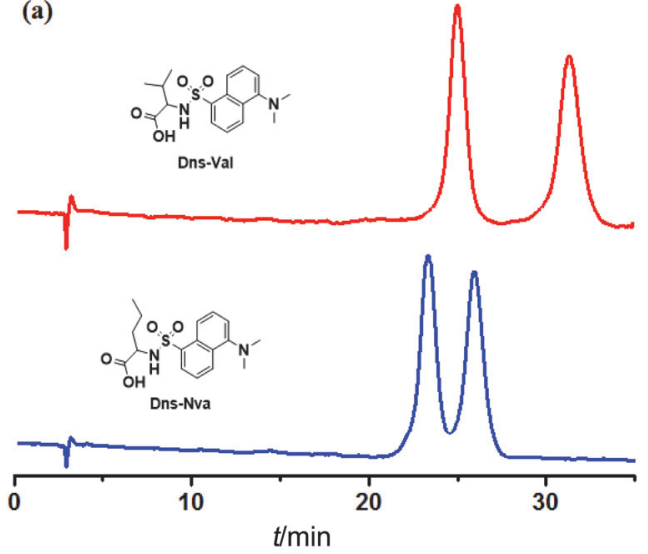

表 1 ANCDCSP 对丹磺酰氨基酸类的分离结果

Table 1 The enantioseparation of dansyl amino acids on ANCDCSP

\begin{tabular}{crrccc}
\hline 样品 & $k_{1}$ & $k_{2}$ & $\alpha$ & $R_{\mathrm{s}}$ & 分离条件 $^{a}$ \\
\hline Dns-Nva & 7.05 & 7.92 & 1.12 & 1.5 & $\mathrm{I}$ \\
Dns-Val & 7.62 & 9.79 & 1.29 & 3.2 & $\mathrm{I}$ \\
Dns-Leu & 10.15 & 13.80 & 1.36 & 3.9 & $\mathrm{I}$ \\
Dns-Glu & 33.84 & 35.36 & 1.04 & 0.4 & $\mathrm{II}$ \\
Dns-Aba & 6.36 & 6.85 & 1.08 & 0.9 & $\mathrm{I}$ \\
Dns-Aca & 10.44 & 13.96 & 1.34 & 3.9 & $\mathrm{I}$ \\
Dns-Phe & 14.59 & 22.42 & 1.54 & 5.3 & $\mathrm{I}$ \\
Dns-Ser & 7.47 & 7.83 & 1.05 & 0.4 & $\mathrm{I}$ \\
Dns-Nle & 7.94 & 9.84 & 1.24 & 2.8 & $\mathrm{I}$ \\
Dns-Thr & 6.44 & 7.14 & 1.11 & 1.3 & $\mathrm{I}$ \\
\hline
\end{tabular}

${ }^{a} \mathrm{I} \mathrm{MeOH} / 1 \%$ TEAA pH $4.10=70 / 30(\mathrm{~V} / \mathrm{V})$, flow rate $0.7 \mathrm{~mL} \cdot \mathrm{min}^{-1}$, temperature: $25{ }^{\circ} \mathrm{C}$. II $\mathrm{MeOH} / 1 \%$ TEAA pH $4.10=60 / 40(V / V)$, flow rate 0.7 $\mathrm{mL} \cdot \min ^{-1}$, temperature: $25{ }^{\circ} \mathrm{C}$.

通过对 Dns-Nva、Dns-Val 与 Dns-Leu、Dns-Nle 两 组样品的横向对比发现，侧链同碳数取代基，支链取代 比直链取代的分离效果好(图 2a), 其保留因子和分离度 都有所增加, 这主要是由于支链烷基相比直链烷基具有 更大疏水体积，在分析物的丹磺酰基团嵌入顶层或底层 环糊精空腔后, 该侧链可与另一环糊精疏水空腔产生立 体作用，提升了手性识别能力，该结果表明手性碳上烷 基链的疏水作用强弱是影响手性拆分效果的一个重要 因素. 对比 Dns-Aba、Dns-Nva、Dns-Nle、Dns-Aca，取 代基上的碳链碳数依次增加, 在相同条件下保留因子和 分离度也依次增加(图 2b), 因为碳数越多疏水作用越强, 同样也说明了手性拆分效果受到手性碳上烷基链疏水 作用的强烈影响. 进一步对比 Dns-Ser 和 Dns-Thr，后者 比前者多一个甲基取代基，增大其疏水性，因此分离度 提高.

(b)

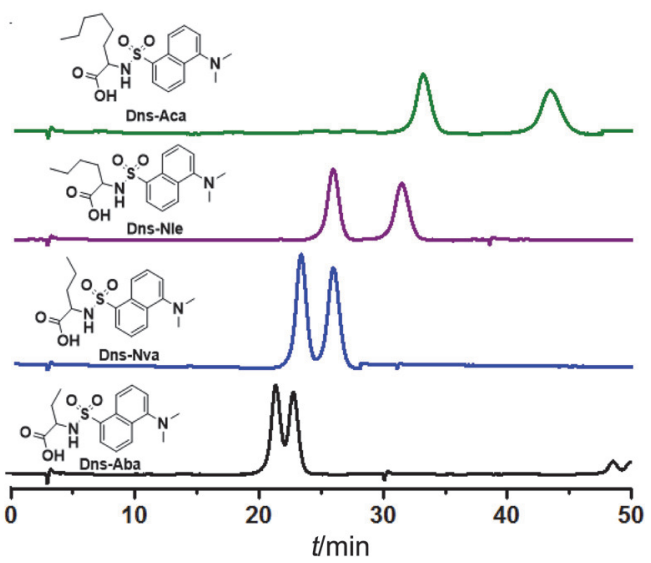

图 2 分析物(a) Dns-Nva、Dns-Val; (b) Dns-Aba、Dns-Nva、Dns-Nle、Dns-Aca 在相同条件下手性拆分对比. 分离条件: 甲醇/三乙胺缓冲液, pH $4.10=70 / 30(V / V)$, 流速 $0.7 \mathrm{~mL} \cdot \mathrm{min}^{-1}, 25{ }^{\circ} \mathrm{C}$

Figure 2 The chiral separation comparisons of (a) Dns-Nva, Dns-Val; (b) Dns-Aba, Dns-Nva, Dns-Nle, Dns-Aca. Separation conditions: $\mathrm{MeOH} / 1 \% \mathrm{TEAA}, \mathrm{pH} 4.10=70 / 30(V / V)$, flow rate $0.7 \mathrm{~mL} \cdot \mathrm{min}^{-1}$, temperature: $25{ }^{\circ} \mathrm{C}$ 
表 2 ANCDCSP 对小分子芳香酸类中的分离结果

Table 2 The enantioseparation results of aromatic acids on ANCDCSP

\begin{tabular}{lccccc}
\hline \multicolumn{1}{c}{ 样品 } & $k_{1}$ & $k_{2}$ & $\alpha$ & $R_{\mathrm{s}}$ & 分离条件 $^{a}$ \\
\hline 2ClPhOPrPA & 8.04 & 8.87 & 1.10 & 1.4 & II \\
2PhButA & 3.04 & - & 1.00 & - & I \\
3ClPOPA & 9.21 & - & 1.00 & - & II \\
2POPA & 7.44 & 8.31 & 1.12 & 2.4 & II \\
TPA & 4.09 & 4.50 & 1.10 & 1.0 & I \\
MDA & 5.59 & 6.95 & 1.24 & 2.5 & I \\
DL-3PhLacA & 4.63 & 6.55 & 1.42 & 4.1 & III \\
4-OHPOPA & 3.58 & 4.12 & 1.15 & 1.9 & III \\
2-OH-2-PPA & 7.62 & 10.6 & 1.40 & 1.9 & I \\
4-OH-MDA & 5.08 & 6.20 & 1.22 & 2.0 & I \\
\hline
\end{tabular}

${ }^{a} \mathrm{I} \mathrm{MeOH} / 1 \%$ TEAA pH $4.10=70 / 30(V / V)$, flow rate $0.7 \mathrm{~mL} \cdot \mathrm{min}^{-1}$, temperature: $25{ }^{\circ} \mathrm{C}$. II $\mathrm{MeOH} / 1 \%$ TEAA pH $4.10=60 / 40(V / V)$, flow rate 0.7 $\mathrm{mL} \cdot \mathrm{min}^{-1}$, temperature: $25{ }^{\circ} \mathrm{C}$. III ACN/1\% TEAA pH $4.10=70 / 30(V / V)$, flow rate $0.7 \mathrm{~mL} \cdot \mathrm{min}^{-1}$, temperature: $25{ }^{\circ} \mathrm{C}$.

相同分离条件下, Dns-Phe 样品的保留时间最长、分 离度最好, 这是由于 Dns-Phe 样品上的丹磺酰基团和苯 环取代基可分别与两个环糊精疏水空腔形成较好的包 合，环糊精层间的更好协同效应显著提升了固定相对 Dns-Phe 的分离能力.

\subsection{2 小分子芳香酸类的手性拆分}

众所周知, 反相分离模式下拆分小分子芳香酸类一 直是环糊精手性固定相的弱项. 因此, 为了考察设计制 备的新型 ANCDCDSP 对小分子芳香酸的拆分效果, 本 文选取了 10 种不同类别的单芳环小分子芳香酸进行色 谱实验, 分离结果列于表 2 中.

ANCDCSP 对大多数小分子芳香酸的分离性能优异 (表 2). 2POPA、MDA、DL-3PhLacA、4-OHPOPA、 2-OH-2PPA、4-OH-MDA 六个样品都可实现基线分离;
3CIPOPA、TPA 实现部分分离, 2PhButA、2DClPhOPrA 则未能实现分离. 2DClPhOPrA、3ClPOPA、2POPA 苯 环上的氯取代基数目依次减少，它们的保留因子大小顺 序 $k_{l}(2 \mathrm{DClPhOPrA})>k_{l}(3 \mathrm{ClPOPA})>k_{l}(2 \mathrm{POPA})$, 苯环 上的氯原子取代可增加其氢键作用，因此导致保留增 加. 但由于取代基的影响导致苯环与环糊精的包合方式 发生改变, 致使其分离效果减弱(图 3a), 而 4-OHPOPA 由于在苯环对位存在羟基，其提供的氢键作用有利于立 体识别, 因而其分离效果与 2POPA 相当. 同样的, 相比 于 MDA 样品, 4-OH-MDA 样品苯环上有一个羟基, 因 此保留时间与分离度相当(图 3c). 以上结果说明, 对于 小分子芳香酸类对映体，氯元素的取代不利于手性拆 分, 而对位差基取代则对其手性识别无明显影响(图 3b). 对比 2-OH-2PPA、TPA、MDA 三个样品的结构, 其支 链上的氢键作用位点即羟基取代基，能与环糊精形成氢 键作用力, 获得较好的分离. 该结果说明环糊精对苯环 的包合能力、氢键等对手性拆分起到决定性的作用. MDA 的分离度要远优于 TPA, 与 2-OH-2-PPA 的分离效 果相当, 说明羟基直接与手性碳相连会显著增强其拆分 效果, 这主要得益于更强的立体效应.

\subsection{3 异噁唑啉类的拆分}

异噁唑啉类化合物是重要的医药和农药中间体. 本 文所选取 5 种异噁唑啉对映体考察固定相的拆分性能 (表 3).

由表 3 可见, $4 \mathrm{MeOPh}-\mathrm{Ph}$ 的保留因子稍高于 $\mathrm{Ph}-\mathrm{Ph}$, 但两者的分离度相当, 说明苯环对位取代不能起到提升 分离效果的作用.

对比 MDOPh-OPr 和 4NPh-OPr，后者分离效果远优 于前者, 说明硝基取代有利于其拆分. 而 4NPh-Py 的分 离度低于 $4 \mathrm{NPh}-\mathrm{OPr}$, 说明较好的分离效果是由硝基取 代苯环及吡咯烷酮共同作用的结果. 硝基的强共轭效应

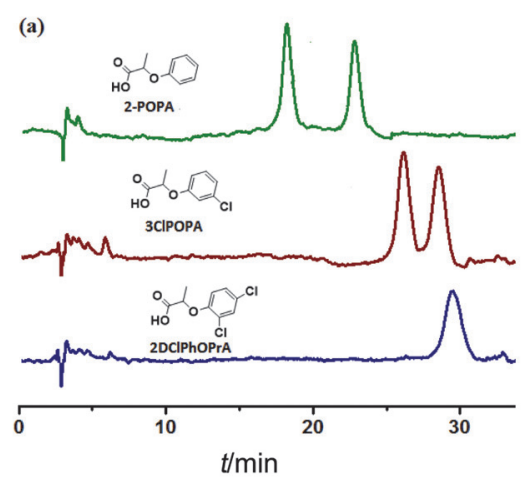

(b)

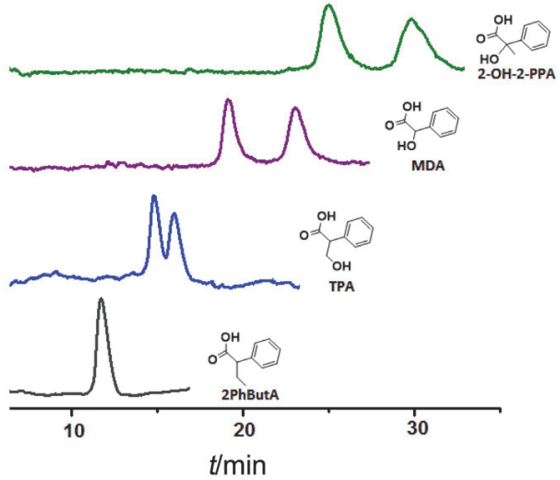

(c)

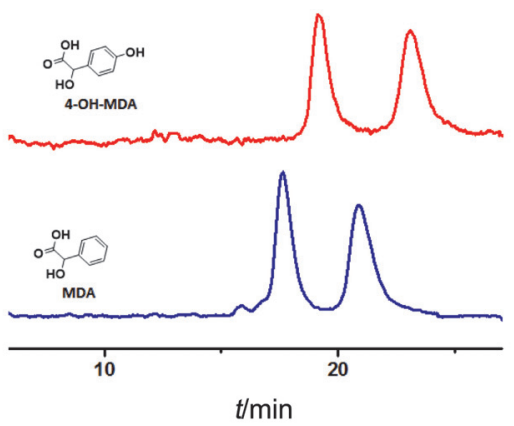

图 3 分析物: (a) 3ClPOPA、2DClPhOPrA、2POPA; (b) 2PhButA、TPA、MDA、2-OH-2-PPA; (c) MDA、4-OH-MDA 的手性拆分对比. [分离条件: (a) 甲醇/三乙胺缓冲液 $\mathrm{pH} 4.10=60 / 40(V / V)$, 流速: $0.7 \mathrm{~mL} \cdot \mathrm{min}^{-1}, 25{ }^{\circ} \mathrm{C}$; (b) 甲醇/三乙胺缓冲液 $\mathrm{pH} 4.10=70 / 30(V / V)$, 流速: $0.7 \mathrm{~mL} \cdot \mathrm{min}^{-1}, 25{ }^{\circ} \mathrm{C}$; (c)乙腈/三乙胺缓冲液 $\mathrm{pH} 4.10=70 / 30(\mathrm{~V} / \mathrm{V})$, 流速: $\left.0.7 \mathrm{~mL} \cdot \mathrm{min}^{-1}, 25{ }^{\circ} \mathrm{C}\right]$

Figure 3 Chiral separation comparisons of (a) 3CIPOPA, 2DCIPhOPrA, 2POPA; (b) 2PhButA, TPA, MDA, 2-OH-2-PPA; (c) MDA, 4-OH-MDA. Separation conditions: (a) $\mathrm{MeOH} / 1 \% \mathrm{TEAA} \mathrm{pH} 4.10=60 / 40(\mathrm{~V} / \mathrm{V})$, flow rate $0.7 \mathrm{~mL} \cdot \mathrm{min}^{-1}$, temperature: $25{ }^{\circ} \mathrm{C}$; (b) $\mathrm{MeOH} / 1 \% \mathrm{TEAA}, \mathrm{pH} 4.10=70 / 30(V / V)$, flow rate $0.7 \mathrm{~mL} \cdot \mathrm{min}^{-1}$, temperature: $25{ }^{\circ} \mathrm{C}$; (c) ACN/1\% TEAA pH $4.10=70 / 30(\mathrm{~V} / \mathrm{V})$, flow rate $0.7 \mathrm{~mL} \cdot \mathrm{min}^{-1}$, temperature: $25{ }^{\circ} \mathrm{C}$. 
使得苯环可较好地与环糊精空腔进行包合, 而吡咯烷酮 羰基可与酯基形成偶极一偶极相互作用, 最终使得 $4 \mathrm{NPh}-\mathrm{OPr}$ 获得最高的分离度.

表 3 ANCDCSP 对异噁唑啉类的分离结果

Table 3 The enantioseparation results of isoxazoline derivatives on ANCDCSP

\begin{tabular}{lccccc}
\hline \multicolumn{1}{c}{ 样品 } & $k_{1}$ & $k_{2}$ & $\alpha$ & $R_{\mathrm{s}}$ & 分离条件 $^{a}$ \\
\hline Ph-Ph & 5.82 & 6.10 & 1.05 & 0.52 & IV \\
4MeOPh-Ph & 6.99 & 7.26 & 1.04 & 0.50 & IV \\
MDOPh-OPr & 2.36 & 2.52 & 1.07 & 0.36 & IV \\
4NPh-OPr & 3.56 & 4.01 & 1.13 & 1.07 & IV \\
4NPh-Py & 7.91 & 8.32 & 1.05 & 0.55 & IV \\
\hline
\end{tabular}

${ }^{a} \mathrm{IV} \mathrm{MeOH} / \mathrm{H}_{2} \mathrm{O}=50 / 50(\mathrm{~V} / \mathrm{V})$, flow rate $0.6 \mathrm{~mL} \cdot \mathrm{min}^{-1}$, temperature: $25{ }^{\circ} \mathrm{C}$.

\subsection{4 其它手性对映体的拆分}

为进一步考察 ANCDCSP 的手性分离性能, 本文选 取了更多手性化合物如苄氟噻嗪(Bendroflumethiazide)、 环氧苯乙烷(Phenyl oxirane)、阿托品(Atropine)、己氨酸 甲基酯 $(D L$-norleucine methyl ester) 以及苯氟雷司 (Benfluorex)等对其进行评价，分离结果列于表 4.

表 4 ANCDCSP 对其它手性对映体的分离效果

Table 4 The enantioseparation results of other enantiomers on ANCDCSP

\begin{tabular}{lccccc}
\hline \multicolumn{1}{c}{ 样品 } & $k_{1}$ & $k_{2}$ & $\alpha$ & $R_{\mathrm{s}}$ & 分离条件 $^{a}$ \\
\hline Bendroflumethiazide & 8.22 & 11.7 & 1.43 & 4.12 & $\mathrm{IV}$ \\
Phenyl oxirane & 0.39 & 0.72 & 1.86 & 1.35 & $\mathrm{IV}$ \\
Atropine & 1.30 & 1.58 & 1.22 & 0.49 & $\mathrm{~V}$ \\
$D L$-norleucine methyl ester & 1.39 & 1.82 & 1.31 & 1.27 & $\mathrm{~V}$ \\
Benfluorex & 2.77 & 3.04 & 1.10 & 0.51 & $\mathrm{~V}$ \\
\hline
\end{tabular}

${ }^{a} \mathrm{IV} \mathrm{MeOH} / \mathrm{H}_{2} \mathrm{O}=50 / 50(\mathrm{~V} / \mathrm{V})$, flow rate $0.6 \mathrm{~mL} \cdot \mathrm{min}^{-1}$, temperature: $25{ }^{\circ} \mathrm{C}$. $\mathrm{V} \mathrm{MeOH} / 1 \%$ TEAA $\mathrm{pH} 4.10=5 / 95(V / V)$, flow rate $0.7 \mathrm{~mL} \cdot \mathrm{min}^{-1}$, temperature: $25{ }^{\circ} \mathrm{C}$.

由表 4 可见，上述化合物在 ANCDCSP 上都可以实 现基线或部分分离, 与我们课题组之前所制备的 DCDCSP 对于 Bendroflumethiazide 样品完全没有拆分的 效果相比, 本文制备的复式环糊精固定相有明显的优势 (图 4). Bendroflumethiazide 的分离度达 4.12, 分析其结
构可知其含有的氧硫双键可与 ANCDCSP 形成较强的 偶极一偶极作用，苯环可与环糊精疏水空腔包合作用， 同时分子中的氨基可与环糊精形成加强络合作用的氢 键作用力, 因此 ANCDCSP 对 Bendroflumethiazide 样品 有较好的分离效果. Phenyl oxirane 样品中的氧杂三元环 中的氧可以与环糊精形成加强络合作用的氢键作用力, 同时苯环可与 ANCDCSP 形成 $\pi-\pi$ 作用, 使得 ANCDCSP 对 Phenyl oxirane 样品也有不错的分离效果. 对于 Atropine、 $D L$-norleucine methyl ester、Benfluorex 三个样品主要是通过偶极一偶极作用以及氢键作用力来 达到分离的效果. Atropine 样品由于分子体积较大使得 络合位阻变大，导致分离效果相对较差. Benfluorex 样 品由于氟取代基增大了芳香环的极性, 使分析物与环糊 精空腔络合减弱，因此分离效果下降. 令人振奋的是对 于没有芳环的 $D L$-norleucine methyl ester, 在该固定相 材料也能取得较好的分离, 主要得益于与手性碳相连的 各基团所提供的偶极-偶极、氢键及疏水作用. 部分代表 性分离图列于图 4 中.

为考察该固定相的稳定性，本文选取几种对映体， 考察固定相对其在不同时期、相同分离条件下的手性拆 分结果. 如图 S4 所示, 尽管色谱柱经过长时间的不同 $\mathrm{pH}(4.1 \sim 8.0)$ 、温度、流动相配比等色谱分离条件, 色 谱柱对对映体的保留及分离结果相差较小, 该结果证明 固定相 ANCDCSP 对本文选取的分离条件具有非常优 异的稳定性. 但由于酯基的水解性，使用该类固定相时 应避免酸性或碱性较强的流动相.

\section{3 与复式天然环糊精固定相(DCDCSP)比较}

我们课题组前期制备了一种复式天然环糊精固定 相(DCDCSP)(结构见图 S5), 实现了多种对映体的分离 并比较了其与单层环糊精固定相材料的优越性 ${ }^{[15]}$. ANCDCSP 与之前制备的未修饰 DCDCSP 手性拆分性 能有一定的差异. 通过对比 ANCDCSP 和 DCDCSP 的分 离结果, 可进一步体现其优势. 表 5 列出了相同的分离 条件下两种固定相对部分分析物分离的对比结果，一些 丹磺酰氨基酸和小分子芳香酸的分离度在 ANCDCSP 上得到显著提高，同时一些 DCDCSP 不能分离的化合
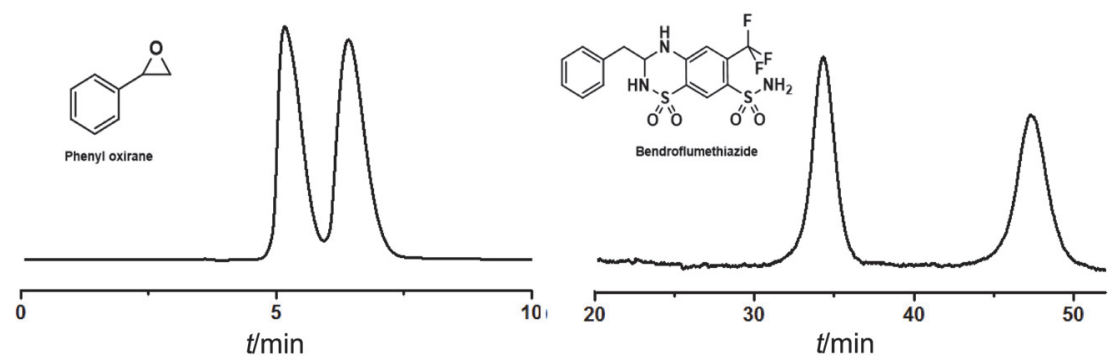

图 4 分析物 Bendroflumethiazide 和 Phenyl oxirane 的手性拆分图. 分离条件: 甲醇 $/ \mathrm{H}_{2} \mathrm{O}=50 / 50(\mathrm{~V} / \mathrm{V})$, 流速: $0.6 \mathrm{~mL} \cdot \mathrm{min}^{-1}, 25^{\circ} \mathrm{C}$

Figure 4 Enantioseparation chromatograms of Bendroflumethiazide and Phenyl oxirane on the ANCDCSP. Separation conditions: $\mathrm{MeOH} / \mathrm{H}_{2} \mathrm{O}=50 / 50$ $(V / V)$, flow rate $0.6 \mathrm{~mL} \cdot \mathrm{min}^{-1}$, temperature: $25{ }^{\circ} \mathrm{C}$ 
物, 如苄氟噻嗪, 环氧苯乙烷等在 ANCDCSP 上都能实 现较好的分离. 这都得益于 $\mathrm{CD}$ 上引入的乙酰基所提供 的氢键, 偶极-偶极相互作用以及立体作用. 相比于天 然复式环糊精结构, 杂化的双层环糊精能提供的多重手 性作用位点增强了其对某些物质的手性识别能力, 同时 也提高了手性分离通量. 但对于多数小分子芳香酸类, ANCDCSP 的分离效果低于 DCDCSP, 可能是由于底层 环糊精羟基的乙酰化减弱了固定相氢键给体的作用导 致的结果.

表 5 ANCDCSP 和 DCDCSP 对一些手性对映体分离的比较

Table 5 Comparison of separation of some chiral enantiomers on ANCDCSP and DCDCSP

\begin{tabular}{llll}
\hline \multirow{2}{*}{ 样品 } & \multicolumn{2}{c}{ DCDCSP } & \multicolumn{2}{c}{ ANCDCSP } & \multicolumn{1}{c}{ 分离条件 $^{a}$} \\
\cline { 2 - 3 } & \multicolumn{3}{c}{$R_{s}$} \\
Dns-Val & 3.09 & 3.2 & I \\
Dns-Leu & 3.43 & 3.9 & I \\
Dns-Aca & 3.29 & 3.9 & I \\
Dns-Phe & 3.88 & 5.3 & I \\
Dns-Nle & 2.51 & 2.8 & I \\
TPA & 0.54 & 1.0 & I \\
Bendroflumethiazide & - & 4.12 & IV \\
Phenyl oxirane & - & 1.35 & IV \\
\hline
\end{tabular}

${ }^{a} \mathrm{I} \mathrm{MeOH} / 1 \% \mathrm{TEAA} \mathrm{pH} 4.10=70 / 30(V / V)$, flow rate $0.7 \mathrm{~mL} \cdot \mathrm{min}^{-1}$, temperature: $25{ }^{\circ} \mathrm{C}$. IV $\mathrm{MeOH} / \mathrm{H}_{2} \mathrm{O}=50 / 50(V / V)$, flow rate $0.6 \mathrm{~mL} \cdot \mathrm{min}^{-1}$, temperature: $25{ }^{\circ} \mathrm{C}$.

本文也比较了 ANCDCSP 与点击反应前单层 CD 固 定相 ACDCSP 的分离效果. 实验发现, ACDCSP 除对 bendroflumethiazide $\left(R_{\mathrm{s}}=1.2\right)$ 、benfluorex $\left(R_{\mathrm{s}}=1.1\right)$ 以及
部分 isoxazolines $\left(R_{\mathrm{S}}<1.0\right)$ 有一定的分离效果外，对其 余分析物均无分离能力. 该结果进一步说明“天然-乙酰 基衍生化”三唑桥联杂化复式环糊精结构在分离能力及 分离通量方面的优势.

\section{3 结论}

本文通过点击化学反应在硅胶表面自下而上地构 建了三唑桥联复式杂化环糊精手性固定相(ANCDCSP), 同时考察了该固定相对不同药物对映体的拆分能力. 相 比于单层的环糊精固定相, 杂化复式环糊精手性固定相 (ANCDCSP)既具有乙酰基修饰的环糊精所提供的偶极偶极及氢键作用(羰基)，又具有天然环糊精的氢键(羟 基)与包合作用，这种协同效应使其对对映体具有更强 的包合作用和手性识别能力. 丹磺酰氨基酸类侧链性质 以及小分子芳香酸类氢键作用在其手性识别过程中起 到举足轻重的作用. 可以预测，本文开发的自下而上基 于协同效应环糊精二聚体新型功能材料的制备方法将 在分离、固相萃取、催化及药物传递方面有重要的应用 潜力.

\section{4 实验部分}

复式环糊精手性固定相(ANCDCSP)的设计合成： 为构建相应的新型复式环糊精手性固定相, 本文采用图 5 所示的的制备路线. 其中, 6 位单取代对甲基苯磺酰 基- $\beta$-环糊精 (TsO-CD)、 6 位单取代叠氮 $-\beta$-环糊精 $\left(\mathrm{N}_{3}-\mathrm{CD}\right)$ 和点击反应催化剂 $\mathrm{CuI}\left(\mathrm{PPh}_{3}\right)$ 依据文献 $[14 \mathrm{~b}]$ 报 道的方法制备.
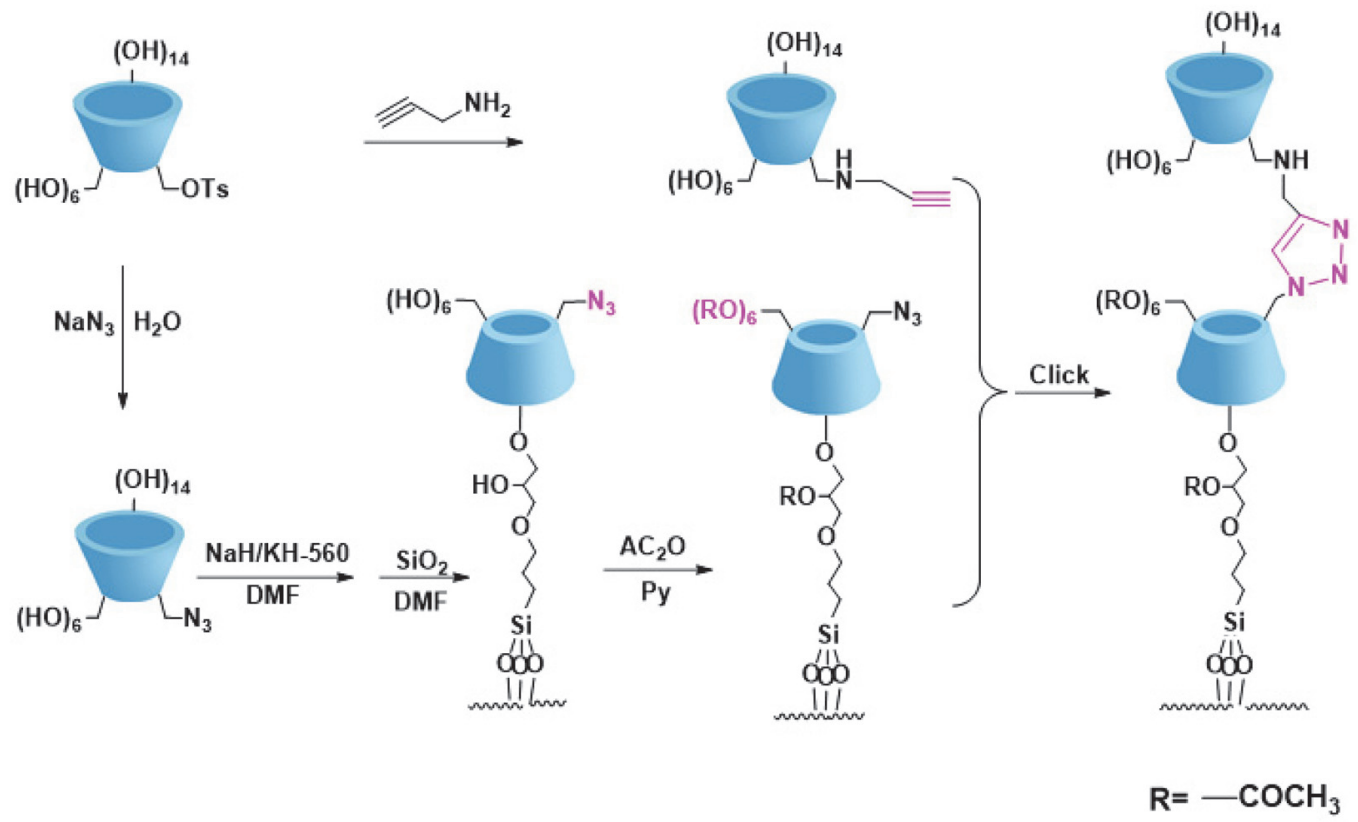

图 5 复式 ANCDCSP 的合成路线

Figure 5 Synthetic pathway of the bilayer CD CSP 


\section{1 炔丙氨基-CD 的制备}

将 TsO-CD (2 g, $1.55 \mathrm{mmol})$ 和炔丙胺 $(0.298 \mathrm{~mL}$, $4.65 \mathrm{mmol}$ )溶于 $5 \mathrm{~mL}$ 无水 $N, N$-二甲基甲酰胺(DMF)中, $80{ }^{\circ} \mathrm{C}$ 下氮气保护搅拌反应 $24 \mathrm{~h}$. 反应停止后, 加入丙 酮 $(20 \mathrm{~mL})$, 析出大量白色固体. 过滤并用丙酮洗涤固体 两次, 真空干燥得浅黄色松散状固体粉末 $2.08 \mathrm{~g}$, 产率 $93 \%$.

\section{$4.2 \mathrm{~N}_{3}-\mathrm{CD}$ 衍生化硅胶 $\left(\mathrm{N}_{3} \mathrm{CDCSP}\right)$ 的制备}

氢化钠 $(259.2 \mathrm{mg}, 6.48 \mathrm{mmol})$ 加入到 $\mathrm{N}_{3}-\mathrm{CD}(6.28 \mathrm{~g}$, $5.4 \mathrm{mmol}$ )(真空干燥)的无水 DMF (130 mL) 溶液中, 在 氮气保护下室温搅拌反应直至不再产生气泡为止. 将上 述溶液过滤, 在滤液中加入(3-环氧丙氧基丙基)三甲氧 基硅烷(1.53 g, $6.45 \mathrm{mmol})$, 该体系在氮气保护下 $90{ }^{\circ} \mathrm{C}$ 搅拌反应 $4 \mathrm{~h}$. 待上述溶液冷却到室温, 加入活化硅胶 $(100 \AA, 5 \mu \mathrm{m})(12 \mathrm{~g}), 110{ }^{\circ} \mathrm{C}$ 下搅拌反应 $24 \mathrm{~h}$. 停止反应 并冷却至室温, 过滤所得固体依次用 DMF、蒸馏水、无 水乙醇、丙酮洗涤, $60{ }^{\circ} \mathrm{C}$ 下真空干燥得到目标产物.

\section{3 乙酰基衍生化(ACDCSP)的制备}

在 $\mathrm{N}_{3} \operatorname{CDCSP}(2.4 \mathrm{~g})$ 的无水吡啶 $(15 \mathrm{~mL})$ 悬浮液中加 入乙酸酎 $(2.5 \mathrm{~g}, 24.5 \mathrm{mmol})$, 氮气保护下 $80{ }^{\circ} \mathrm{C}$ 搅拌反 应 $19 \mathrm{~h}$. 抽滤, 所得固体分别用无水乙醇 $(50 \mathrm{~mL})$ 、丙酮 $(40 \mathrm{~mL})$ 洗涤后真空干燥 $1 \mathrm{~h}$, 得到白色粉末 $2.4 \mathrm{~g}$.

\subsection{ANCDCSP 的点击制备}

将炔丙氨基-CD (1.2 g, $0.88 \mathrm{mmol}$ )溶解于无水 DMF $(30 \mathrm{~mL})$ 中, 依次在上述溶液中加入 $\mathrm{CuI}\left(\mathrm{PPh}_{3}\right)(9 \mathrm{mg}, 0.5$ mmol\%)、ACDCSP (2.3 g), $90{ }^{\circ} \mathrm{C}$ 下氮气保护反应 $2 \mathrm{~d}$. 反应停止后冷却至室温, 过滤, 所得固体依次用 DMF 洗涤 2 次, 然后再用丙酮索式提取 $8 \mathrm{~h}$, 真空干燥得目标 产物.

\section{References}

[1] Zhao, L.-Z.; Zhong, G.-P.; Huang, M. Chin. J. Pharm. Anal. 2005, 25,1203 . (赵立子, 钟国平, 黄民, 药物分析杂志, 2005, 25, 1203.)

[2] Wu, X.-D.; Zhu, Y.-E. Chin. J. Pharm. Anal. 2008, 28, 715. (吴澓 丹, 朱亚尔, 药物分析杂志, 2008, 28, 715.)

[3] Yang, L.-Q.; He, N.; Zhang, Y.-B. Chin. J. Med. Chem. 2000, 9, 817. (杨柳青, 何南, 张玉彬, 中国新药杂志, 2000, 9, 817.)

[4] Matthews, S. J.; McCoy, C. Clin. Thera. 2015, 25, 342.

[5] Pérez Olivero, S. J.; Pérez Trujillo, J. P. J. Agric. Food Chem. 2010, $58,12976$.

[6] (a) Saavedra, L.; Nickerson, B.; Borjas, R. E.; Lynen, F.; Sandra, P. J. Chromatogr. B 2008, 875, 248; (b) Zhang, J.; Du, Y.; Zhang, Q. J. Chromatogr. A 2013, 1316, 119; (c) Yan, T. Q.; Orihuela, C. J. Chromatogr. A 2007, 1156, 220.

[7] (a) Tong, S.; Zhang, H.; Shen, M.; Ito, Y.; Yan, J. Z. J. Chromatogr. B 2014, 962, 44. (b) Gua, J.; Yang, J.; Bi, Y. J. Sep. Sci. 2008, 31 288; (c) Zhou, R.-D.; Li, L.-S.; Chen, B.-P.; Nie, G.-Z.; Zhang, H.-F. Acta Chim. Sinica 2014, 72, 720. (周仁丹, 李来生, 程彪平, 聂桂 珍, 张宏福, 化学学报, 2014, 72, 720.)

[8] Armstrong, D.; Ward, T.; Armstrong, R. Science 1986, 232, 1132.

[9] (a) Zhong, Q.; He, L.; Beesley, T. E. J. Chromatogr. A 2006, 1115 , 19. (b) Soukup, R. J.; Rozhkov, R. V.; Larock, R. C. Chromatographia 2005, 61, 219. (c) Wang, R.-Q.; Ong, T.-T.; Tang, W. Anal. Chim. Acta 2012, 718, 121.

[10] (a) Zhao, J.; Lu X.-H.; Wang, Y.; Lv, J. J. Chromatogr. A 2015, 1381, 253. (b) Takashima, Y.; Yuting, Y.; Otsubo, M; Yamaguchi, H.; Harada, A. J. Org. Chem. 2012, 8, 1594. (c) Watanabe, K.; Kitagishi, H.; Kano, K. Angew. Chem., Int. Ed. 2013, 52, 6894.

[11] (a) Blaszkiewicz, C.; Bricout, H.; Léonard, E. Chem. Commun. 2013, 49, 6989. (b) Kolb, H. C.; Finn, M. G.; Sharpless, K. B. Angew. Chem., Int. Ed. 2001, 40, 2004.

[12] (a) Wang, Y.; Young, D. J. J. Chromatogr. A 2010, 1217, 7878; (b) Wang, H.-S.; Pen, J.-T.; Wei, J.-P.; Jiang, A. Acta Chim. Sinica. 2012, 12, 1355. (王怀松, 彭江涛, 魏纪平, 姜安, 化学学报, 2012, 70, 1355.)

[13] (a) Guo, Z.; Jin, Y.; Liang, T. J. Chromatogr. A 2009, 1216, 257. (b) Wang, Y.; Young, D. J.; Ng, S. C. J. Chromatogr. A 2010, 1217, 5103.

[14] (a) Wang, Y.; Ong, T.-T.; Li, L.-S. J. Chromatogr. A 2009, 1216, 2388. (b) Wang, Y.; Chen, H.; Xiao, Y.; Ng, C. H.; Oh, T. S.; Tan, Y. T. T.; Ng, S. C. Nat. Protocols 2011, 6, 935.

[15] Zhao, J.; Lu, X.-H.; Wang, Y. J. Chromatogr. A 2014, 1343, 101.

(Qin, X.) 